\title{
Modeling Weak Fluctuations of Undersea Telemetry Signals
}

\author{
Timothy F. Duda \\ ( Invited Paper)
}

\begin{abstract}
Amplitude and phase fluctuations of monochromatic acoustic signals in the ocean can be predicted by calculating statistical properties of wave equation perturbation solutions. For ranges less than a few kilometers in an active mixing region, or less than $15 \mathbf{~ k m}$ in the deep ocean, weak fluctuation theory can be used to model signal fluctuations in frequency and spatial frequency. Fluctuation predictions for transmission through realistic vector wavenumber spectra of oceanic sonic refractive index fluctuations are evaluated numerically and help define the ocean regions amenable to telemetry.

Keywords - Wave propagation, forward scattering, internal waves, microstructure.
\end{abstract}

\section{INTRODUCTION}

NHOMOGENEITIES of sound speed in the ocean caused by Linternal waves, layering, and turbulence induce fluctuations in acoustic transmission. Sound waves from a point source are perturbed from simple spherical waves into complicated phase fronts, including multipath, resulting in random patterns of signal phase and amplitude in both time and space.

In previous works, Rytov scattering theory and realistic vector wavenumber spectra of small-scale ocean sound speed structure were used to predict spatial spectra of signal fluctuations over short propagation ranges [1]-[3]. Measurement of ocean turbulence and fine structure parameters requires measurement of spatial gradients from which acoustic fluctuations arise, therefore using acoustics for monitoring ocean turbulence was investigated. Most preceding work utilized less realistic [4] or less comprehensive [5] ocean models to predict weak-fluctuation propagation parameters.

Over short ranges, characterized well by a single transmission path from a source to any receiver, the wave front may be subject to effects weak enough that the variation of signal amplitude in a plane transverse to the transmission path, or the variation in time, is somewhat less than the average amplitude. This is the regime where perturbation solution of the wave equation in the random medium provides useful results [6]-[9]. The Born and Rytov perturbation solutions for the wavefield have been referred to as single-scattering solutions, because the incident wavefield at all ranges between the source and receiver is taken to be unaltered from its form in the homogeneous medium, and the resultant contribution to the total field reflects only inhomogeneities at that range. They are intuitively inappropriate where the resultant field is significantly altered from the

Manuscript received August 23, 1990; revised October 12, 1990. This work was supported by the Office of Naval Research, Ocean Acoustics Program. The computing resources were partially supported by the $\mathrm{W}$. M Keck Foundation. This work represents Institute of Tectonics Contribution No. 114.

The author is with the Institute of Tectonics and Institute of Marine Sciences, University of California, Santa Cruz, CA 95064

IEEE Log Number 9041228 unscattered (homogeneous medium) form. In those situations, multiple-scattering solutions are in order (e.g., [10], [11]). Using perturbation theory to study ocean propagation at short ranges is simpler than using the multiple-scattering theories, because it requires no special properties of the medium and because it can handle long-range modulations of random medium heterogeneities (due to the independent contributions at each range).

The Rytov solution, or the method of smooth perturbations, has been shown to accurately describe wave transmission when the normalized variance of intensity $\sigma_{I}^{2}$ (sometimes called the scintillation index) is less than 0.3 [8], [12], [13]. That is, when

$$
\sigma_{I}^{2} \equiv \frac{\left\langle I^{2}\right\rangle-\langle I\rangle^{2}}{\langle I\rangle^{2}}<0.3 .
$$

Accounts of the Rytov technique are found in Tatarskii [6], Fante [12], and Goodman [14, chap. 8]. Clifford [7] and Strohbehn [8] review the history of the Born and Rytov perurbation solutions and the unsuccessful attempts to extend them beyond their regions of validity. One interesting point is that the phase fluctuations predicted with perturbation methods may be accurate beyond the above-stated limit for intensity fluctuation predictability. This was alluded to for laser propagation in the atmosphere [7], and demonstrated in the ocean for $\sigma_{I}^{2} \approx 0.7$ [15].

In the Rytov solution, spectra or covariance functions of log-amplitude or phase fluctuations in a transverse observing plane for signals from a point source are known functions of the vector wave-number spectrum of sound speed fluctuations in the intervening medium. Using model media characteristic of the ocean, we calculate transverse fluctuation spectra in an observing plane and temporal fluctuation spectra at single points. Integrals of these yield scintillation indices or rms phase fluctuations, obtainable from arrays or from experimental time series that cover many characteristic time scales for the medium fluctuations or have fluctuations due to a medium advecting quickly past. Expressions for transverse spectra are outlined in Section II, and for temporal spectra in Section III. Model sound speed fluctuation spectra are described in Section IV.

Our model media have anisotropic fine-scale sound speed fluctuations and isotropic microscale (less than $1 \mathrm{~m}$ ) fluctuations. Medium fluctuation spectra are all band-limited analytic expressions. We present spectra for vertical and nearly horizontal transmission.

In general, finescale fluctuations contribute more to signal variability than the less anisotropic (and more intermittent) microscale fluctuations. For near-horizontal transmission in the ocean, the transverse spectrum is anisotropic because of the anisotropy of the intervening finescale ( 1 to $100 \mathrm{~m}$ vertical size) sound speed fluctuations, with the typical scale length of inten- 
sity fluctuations being much smaller in the vertical. The transverse intensity spectrum should be isotropic for vertical transmission, since only microstructure is expected to produce intensity fluctuation.

Limits of applicability of unsaturated theory in frequencyrange coordinates are a general result of the calculations. Another general result is that the effect of any microstructure is substantially increased with increasing frequency, so that the relative effects of finestructure and microstructure can be unraveled if a combination of frequencies and ranges is used. Fine structure and microstructure effects can be evaluated simultaneously and unambiguously if the proper frequencies and ranges are used. These and other generally applicable results are presented in Section V.

\section{Transverse Acoustic Fluctuations}

Spherical-wave signals from a point source with frequency $\sigma$ propagating through a medium of randomly fluctuating wave speed,

$$
c(x)=C_{0}(1+\mu(x)), \quad\langle\mu\rangle=0
$$

are governed by the Helmholtz equation:

$$
\nabla^{2} \psi+q^{2}\left[\frac{C_{0}}{c(\boldsymbol{x})}\right]^{2} \psi=0
$$

where $q=\sigma / C_{0}$ is a reference acoustic wave number, and $\psi(\boldsymbol{x})$ is the demodulated, single-frequency acoustic pressure wave function. The Rytov method gives a solution for perturbation log-amplitude and phase, defined by

$$
\psi \equiv \psi_{0} A e^{i \phi}
$$

where $\psi_{0}$ is the field for $\left(c=C_{0}\right)$. In addition to the original derivation of the solution (reviewed in [9], [12], [14]), a new method has appeared [16], [17]. Statistics of $A$ and $\phi$ can be computed directly from the solution, as well as statistics of perturbation log-intensity, given by

$$
\iota \equiv 2 \ln A=\ln (I /\langle I\rangle)
$$

where $\iota+1 \approx I /\langle I\rangle$ in the region of validity.

For nearly horizontal propagation angles (less than $15^{\circ}$ or so), the random field $\mu$ may also include an additional linear vertical gradient term which will have no effect on fluctuation solutions and can be disregarded. This term can approximately represent nonaxial portions of a sound channel. This allows application of the Rytov solution to horizontal transmission in the main thermocline, for example.

Starting with covariance functions, we compute one-dimensional spectra of $\log$-intensity, $\log$-amplitude, and phase variability. For sound speed fluctuations $\mu$ in the medium (also referred to as refractive index perturbations) characterized by a spatial spectrum, disregarding slowly varying deterministic effects of the sound channel or waveguide, the Rytov expressions for covariance functions in a transverse observing plane are

$$
\begin{aligned}
C(r, R)= & 2 \pi q^{2} \int_{0}^{R} d x_{1} \int_{-\infty}^{\infty} d k_{x_{2}} d k_{x_{3}} F\left(0, k_{T}\right) \\
& \times g\left[\frac{x_{1}\left(R-x_{1}\right)}{2 q R}\left(k_{x_{2}}^{2}+k_{x_{3}}^{2}\right)\right] \exp \left(i k \cdot r x_{1} / R\right)
\end{aligned}
$$

where

$$
C(r, R, q) \equiv\left\langle\beta\left(r_{0}, R, q\right) \beta\left(r_{0}+r, R, q\right)\right\rangle
$$

and where $\beta$ can be either $A$ or $\phi$. Transmission is in the $x_{1}$ direction over a range $R . F(k)$ is the vector spectrum of sound speed fluctuations, so that $\left\langle\mu^{2}\right\rangle=d^{3} \boldsymbol{k} F(\boldsymbol{k})$, with $\boldsymbol{k}=$ $\left(k_{x_{1}}, \boldsymbol{k}_{T}\right)=\left(k_{x_{1}}, k_{x_{2}}, k_{x_{3}}\right)$ and $k=|\boldsymbol{k}|$. The function $g$ is sine $^{2}$ if $\beta=A$, and is cosine ${ }^{2}$ if $\beta=\phi$. Unsaturated scattering effects are maximum for media fluctuations at the Fresnel radius, $R_{f}=(R \lambda)^{1 / 2}$. The paraxial approximation, which has been invoked, requires that scattering be limited to small angles, or equivalently that the parabolic wave equation be valid. The log-intensity covariance is four times log-amplitude covariance. We consider log-intensity for the remainder of this section.

Introduce cartesian coordinates $x, y, z$ tied to the medium, with $z$ upward. For horizontal propagation to a transverse plane, set $x_{1}=x, x_{2}=y, x_{3}=z$ and $r=z$, so that the vertical wave number power spectrum of $\iota$ is defined by

$$
\Theta(m ; R, q)=\frac{1}{2 \pi} \int_{-\infty}^{\infty} d z C(z, R) e^{-i m z} .
$$

Substitution of (6) into (8), followed by integration over $z$ and $k_{z}$, yields:

$$
\begin{aligned}
& \Theta(m ; R, q) \\
& =8 \pi R q^{2} \int_{0}^{R} \frac{d x}{x} \int_{-\infty}^{\infty} d k_{y} F\left(0, k_{y}, k_{z}=m R / x\right) \\
& \quad \times \sin ^{2}\left[\frac{x(R-x)}{2 q R}\left(k_{y}^{2}+\frac{m^{2} R^{2}}{x^{2}}\right)\right] .
\end{aligned}
$$

Scaling out the range, $s=x / R$,

$$
\begin{aligned}
\Theta(m ; R, q) & \\
= & 8 \pi R q^{2} \int_{0}^{1} \frac{d s}{s} \int_{-\infty}^{\infty} d k_{y} F\left(0, k_{y}, k_{z}=m / s\right) \\
& \times \sin ^{2}\left[\frac{s R(1-s)}{2 q}\left(k_{y}^{2}+m^{2} / s^{2}\right)\right] .
\end{aligned}
$$

Since $R_{f}^{2}=2 \pi R / q$, the integral is a function of $R_{f}$, but not of $R$ or $q$ independently. Scaling the horizontal wave number $l=k_{y} s / m$ and substituting a scaled wave number $m_{f}=R_{f} m$, one finds:

$$
\begin{aligned}
\Theta(m)= & 8 \pi R q^{2} \int_{0}^{1} d s \frac{m_{f}}{R_{f} s^{2}} \int_{-\infty}^{\infty} d l \\
& \times F\left(0, k y=\frac{l_{f}}{R_{f} s}, k_{z}=\frac{m f}{R_{f} s}\right) \\
& \times \sin ^{2}\left[\frac{m_{f}^{2}}{4 \pi s}(1-s)\left(l^{2}+1\right)\right] .
\end{aligned}
$$

The intensity fluctuations observed at wave number $m$ have contributions from medium fluctuations of different vertical wave numbers $k_{z}=m / s$, dependent on the range from the source.

For propagation at angle $\xi$ from the horizontal, a coordinate transformation can be made:

$$
\begin{aligned}
k_{x_{1}} & =k_{x} \cos \xi+k_{z} \sin \xi \\
k_{T} & =\left(k_{y}, k_{z} \cos \xi-k_{z} \sin \xi\right) .
\end{aligned}
$$


Rather than evaluating $F\left(k_{x}=0, \cdots\right)$ as in (11), we substitute the general form:

$$
F\left(k_{x_{1}}=0, k_{x_{2}}=\frac{l m_{f}}{R_{f} s}, k_{x_{3}}=\frac{m_{f}}{R_{f} s}\right) .
$$

If $\tan (\xi) \ll 1$, this simplifies to

$$
F\left(k_{x}=k_{z} \tan \xi, k_{y}=\frac{l m_{f}}{R_{f} s}, k_{z}=\frac{m_{f}}{R_{f} s}\right) .
$$

For vertical propagation, (11) may be used after rotation (exchange of $k_{z}=0$ for $k_{x}=0$ and $k_{x}$ for $k_{z}$ ).

There is a useful scaled form of the spectrum for the fine-scale models we consider. The constant $R_{f}$ can be removed from the integral if $F(k)$ has the form $\boldsymbol{k}^{-p}$, in which case one can write:

$$
\begin{aligned}
\Theta(m)= & R_{f}^{p-1} 8 \pi R q^{2} \int_{0}^{1} d s \frac{m_{f}}{s^{2}} \int_{-\infty}^{\infty} d l \\
& \times F\left(0, k_{y}=\frac{l m_{f}}{s}, k_{z}=\frac{m_{f}}{s}\right) \\
& \times \sin ^{2}\left[\frac{m_{f}^{2}}{4 \pi s}(1-s)\left(l^{2}+1\right)\right] .
\end{aligned}
$$

The integral is a function of $m_{f}$ only and is called $\Theta_{f}$. The spectrum

$$
\Theta_{f}\left(m_{f}\right)=\frac{\Theta(m)}{R_{f}^{p-1} 8 \pi R q^{2}}
$$

is independent of $R, q$, and $R_{f}$. Therefore $\Theta_{f}$ can be used to directly compare or average together data from different unsaturated conditions if the power law medium model is appropriate for all cases. This is not true for band-limited power-law spectra, since outer (large) scale or inner scale roll-offs will influence $\Theta$ from experiments with different $R_{f}$ in different ways. It is very nearly true, however, if $R_{f}$ is well within the wavelength band described by the power law. For ocean fine-scale models with outer scales greater than $100 \mathrm{~m}, R_{f}$ must be in this band for the unsaturated approximation to hold. Therefore the $\Theta_{f}\left(m_{f}\right)$ representation can be used with our fine-scale models.

\section{Temporal Acoustic Fluctuations}

Temporal fluctuations arise from two effects: Advection of a "frozen field" of refractive index fluctuations past the apparatus by currents, and evolution of the random index fluctuations. Currents can be incorporated into the modeling and can be extracted from measurements if time evolution of the medium is insignificant. Temporal spectra of ocean internal waves can be modeled using linear dispersion, and temporal spectra of turbulence can be approximated if one gives a time scale (related to strain rate) to each eddy scale [18].

The covariance functions can be generalized to include transverse currents $U(x)[6]$ :

$$
\begin{aligned}
C(\boldsymbol{r}, \tau, R)= & 2 \pi q^{2} \int_{0}^{R} d x_{1} \int_{-\infty}^{\infty} d k_{x_{2}} d k_{x_{3}} F\left(0, k_{T}\right) \\
& \times g\left[\frac{x_{1}\left(R-x_{1}\right)}{2 q R}\left(k_{x_{2}}^{2}+k_{x_{3}}^{2}\right)\right] \\
& \cdot \exp \left(i k\left[r x_{1} / R-U(x) \tau\right]\right) .
\end{aligned}
$$

Farmer et al. [19] used this to estimate the current and turbulence outer scale within a coastal channel of $34 \mathrm{~m}$ maximum depth containing strong (up to $1 \mathrm{~m} / \mathrm{s}$ ), turbulent tidal currents. They transmitted maximal-sequence codes having an $86 \mathrm{kHz}$ center frequency for the horizontal distance $660 \mathrm{~m}$, and observed scintillation indices up to about 0.6 , slightly above the weak fluctuation regime. Their accurate estimates of the current show the validity of the theory.

Evaluation of the time-space covariance functions at zero spatial lag gives the temporal covariance functions. If these are transformed to spectra, a Dirac delta function factor remains, showing an ambiguity between frozen-field advection and time dependence of the inhomogeneities.

If the effect of the current $U$ is disregarded, transformation of fluctuations from the spatial domain to the time domain can be made for internal-wave finestructure. The linear internal-wave dispersion relation is required. For phase and amplitude, the Rytov-type spectra are [3]

$$
\begin{aligned}
\Theta_{\omega}(\omega, j, R)= & 2 q^{2} \int_{0}^{R} d x_{1} k_{x_{2}}^{-1} F(\omega, j ; z) \\
& \times g\left\{\frac { ( j \pi / N _ { 0 } B ) ^ { 2 } } { 2 q } \left[\frac{x_{1}\left(R-x_{1}\right)}{R}\right.\right. \\
& \cdot\left[\omega^{2}-\left(f^{2}+N(z)^{2} \tan ^{2} \xi\left(x_{1}\right)\right)\right] \\
& \left.\left.+N(z)^{2} A_{i}\right]\right\} .
\end{aligned}
$$

The new symbols are the internal wave vertical mode number $j$, buoyancy frequency profile $N(z)$, index buoyancy frequency $N_{0}$, scale-length $B$ of the function $N(z)$, and inertial frequency $f$. There are discussed in [3], [16], [17], [20] and also in the next section. The $A_{i}$ term is the inverse of the phase curvature function [16], [17], which accounts for deterministic sound channel effects and can be included in a more general derivation of (9). Calculations of this type have been compared to ocean data [3], [15].

\section{Model Media $F(\boldsymbol{k})$}

We use two types of models: One characterizes anisotropic, internal-wave-induced, fine-scale sound-speed fluctuations. The other characterizes isotropic microscale fluctuations, but can be extended to larger scales and used to model scintillations through turbulent flows [19]. The fine-scale model allows use of $\Theta_{f}\left(m_{f}\right)$ scaling, with $p=4$. The microstructure models have outer scales near $R_{f}$ and therefore do not collapse to the same $\Theta_{f}\left(m_{f}\right)$ for all $R_{f}$, and also do not have $p=4$, so that results form the microstructure must be analyzed separately for each range and frequency.

Any linear combination of these $F(\boldsymbol{k})$ is also a model, so that microscale and fine-scale effects can be modeled separately for study and then combined for a more complete result.

The fine-scale models are Garrett-Munk ocean internal-wave spectra in sound speed fluctuation form [21], [16], [17]. The fluctuations in sound speed are caused by internal-wave vertical straining of a smooth background sound-speed gradient, so that $\mu_{\mathrm{rms}}$ is related to the rms particle displacement and gradient. The expression is

$$
F_{g}(k, z)=\frac{\phi_{g} k_{h}\left|k_{z}\right|}{\left(k_{h}^{2}+(f / N(z))^{2} k_{z}^{2}\right)^{2}\left(k_{z}^{2}+k_{g}^{2}\right)}
$$


where

$$
\phi_{g}=\left\langle\mu_{0}^{2}\right\rangle\left(\frac{N(z)}{N_{0}}\right)^{2}\left(\frac{2}{\pi^{3}}\right)\left(\frac{f}{N(z)}\right) k_{g}
$$

and where $\left\langle\mu_{0}^{2}\right\rangle$ is the refractive index variance at a depth having buoyancy frequency $N_{0}$, which is simply a reference frequency, and $f$ is the local inertial frequency. The vertical density gradient, which supports the gravity-wave oscillations, is expressed in terms of buoyancy frequency $N(z)$. The spectra are horizontally isotropic, anisotropic in $k$ (note the $(f / N)^{2}$ factor in the denominator), and vertically symmetric. The vertical wave number bandwidth is $k_{g}=j *\left(\pi N(z) / N_{0} B\right)$, with $j *$ the characteristic mode number, and $B$ the vertical length scale of the function $N(z)$. The buoyancy frequency $N(z)$ must vary slowly enough in the vertical so that WKB scaling of vertical modes holds. This allows use of linear internal wave dispersion in the formulation of the vector spectrum. Internal waves in coastal environments may be modeled by adjusting the Garrett-Munk model [1], but many GM features such as horizontal isotropy and vertical symmetry seem unlikely. The variability of forcing and response in those environments precludes a discussion here of any particular coastal internal wave model.

Microscale (order of $1 \mathrm{~m}$ or less) sound speed fluctuations are modeled with a Kolmogorov-type isotropic inertial-convective scalar subrange spectrum [6], [22]. A high-pass filtering with outer scale $k_{t}$ is used in order to confine the subrange to $k_{t}<k$, and a diffusive rolloff $R_{b}$ from the Batchelor spectrum below is included, giving:

$$
F_{k}(k)=\frac{\phi_{t}}{\left(k^{2}+k_{t}^{2}\right)^{11 / 6}} \frac{k^{2}}{k^{2}+k_{t}^{2}} R_{b}(k) .
$$

This model is most sensible in a weakly stratified region such as a surface mixed layer. Ocean stratification generally limits this subrange to scales of less than $1 \mathrm{~m}[23]-[25]$. Sound speed structure at scales smaller than the viscous cutoff scale $l_{c}=$ $\left(v^{3} \epsilon^{-1}\right)^{1 / 4}$ is poorly modeled by $F_{k}(v$ is the kinematic viscosity and $\epsilon$ is the rate of dissipation of kinetic energy). We use this spectrum as a means of adding microscale fluctuations at wave numbers near $R_{f}^{-1}$ to the fine-scale field. Its precise form in the rolloff region is not relevant, since microstructure of scales $R$ or larger contribute dominantly to acoustic fluctuations in the weak fluctuation regime. Modeling of fast turbulent (coastal or tidal) currents or surface-mixing layers can be accomplished by enlarging the outer scale from meters, appropriate for stratified ocean turbulence, to the depth of the channel or mixing layer.

To choose appropriate microstructure levels, we choose $\phi$ such that wave-number magnitude spectra $E_{k}(k)=2 \pi k^{2} F_{k}(k)$ match the Batchelor spectrum $E_{b}(k)$ at a high wavenumber. The spectrum $E_{b}(k)$ is from the theory of passive scalar gradients at subviscous scales (advected by homogeneous isotropic turbulence) [26], with diffusivity of the quantity less than that of momentum (the kinematic viscosity). The Batchelor spectrum $E_{b}$ is applicable for wave numbers greater than $l_{c}^{-1}$ (order of 10 $\mathrm{cpm}$ ), and consists of a viscous-convective subrange and a diffusive rolloff. For a scalar with diffusivity $k$ and with an average least-principal-rate of strain $\gamma=-q_{b}^{-1}(\epsilon / v)^{1 / 2}$, the spectrum is

$$
E_{b}(k)=\frac{-\chi}{\gamma k} R_{b}(k)
$$

with rolloff

$$
R_{b}(k)=\exp \left[\frac{\kappa}{\gamma} k^{2}\right]
$$

where $\chi=2 \kappa \overline{(\nabla a)^{2}}$ is the dissipation rate of the scalar quantity $a$ (either sound speed or temperature, which we relate directly in this work with a disregard of salinity effects for the mid-latitude ocean [17]). Based on upper-ocean observations, we set $q_{b}=3.7$ [27].

Relationships of internal-wave kinetic energy, stratification, $\left\langle\mu^{2}\right\rangle, \chi$, and $\epsilon$ are discussed in [1], [17].

\section{Deep Ocean Transmission}

A few general results can be obtained by calculation of $\Theta_{f}$ using the GM79 version of the Garrett-Munk internal-wave model [20] and various levels of microstructure. In the GM79 model, $N_{0}=3 \mathrm{cph}, B=1300 \mathrm{~m}, j *=3$, latitude is $30^{\circ}$, and $\left\langle\mu_{0}^{2}\right\rangle=2.5 \times 10^{-7}$. Effects of variable transmission angle, wave energy, stratification, and salinity are discussed here.

The GM fine-structure models, since they have essentially power-law forms, scale with frequency and range. Integrating the definition of $\Theta_{f}\left(m_{f}\right)$ over $d m_{f}$ and substituting $R_{f} d m=$ $d m_{f}$ into the $\Theta$ integral, one sees that $\left\langle\iota^{2}\right\rangle=\int \Theta(m) d m$ scales with $R_{f}^{p-2} R q^{2}$. For the GM model, $p=4$, so $R_{f}^{p-2} \propto$ $R / q$. Therefore $\left\langle\iota^{2}\right\rangle \propto q R^{2} \propto \sigma R^{2}$. This is a fundamental scaling that is useful for fluctuation prediction once a stationary medium spectrum is characterized.

In $\Theta$ simulations, 96-point Gaussian integration is used to evaluate (11). Integration limits are $s=[0,1]$ and $l=$ $[-1000,1000]$. After integration for a selection of wavenumbers $m$, the resulting spectrum can be integrated to yield $\sigma_{I}^{2}$ or variances of $A$ or $\phi$. Complete validity of the paraxial approximation requires that acoustic wavenumber times the Fresnel radius be large with respect to the square of the molecular dissipation-controlled cutoff scale (about $2 \mathrm{~cm}$ ): $\lambda R_{f} \ll l_{0}^{2}[6]$. Validity of the calculations can be checked by eliminating medium fluctuations smaller than an artificially large cutoff (inner) scale in a test run and comparing with results using the full medium. Since the ocean models taper off at a high wavenumber, the contribution from the high wavenumber portion, beyond the calculated validity region, does not change the character of the calculations.

It has been suggested that the $\sin ^{2}$ term in (9) is not correct if the correlation length $L$ of medium perturbations along the transmission direction is much greater than $R$, with an alternate result of $\left\langle\iota^{2}\right\rangle \propto \sigma^{3 / 2} R^{5 / 2}$ [28]. However, transmission over ranges of less than $1 \mathrm{~km}$ in the Kane Basin, north of Greenland, gives modest support for the $\left\langle\iota^{2}\right\rangle \propto \sigma R^{2}$ scaling [1]. The value $L \approx 5 \mathrm{~km}$ has been used successfully to predict fluctuations from internal waves for $300 \mathrm{~km}$ transmission [29], [30], but a lower value is appropriate for other angles [30].

Limits of applicability of our technique to transmission in the deep mid-latitute ocean are estimated by calculating the intensity variance in the band $[0.01-10] \mathrm{rad} \mathrm{m}^{-1}$ using the GM79 model. Figs. 1-3 show the region of the $\sigma-R$ domain where the intensity variance is less than 0.3 for 9 situations: $\xi=0^{\circ}$ (horizontal transmission), $\xi=10^{\circ}$, and $\xi=20^{\circ}$ for three different stratification levels, $N(z)=N_{0}, N(z)=N_{0} / 3$, and $N(z)=2 N_{0}$. Unsaturated transmission is seen to extend to the smallest range at $\xi=0$ for given $N$ and $\sigma$, since vertical acoustic wave crests are perpendicular to dominant anisotropic (flattened) internal wave sound-speed structures, leading to a corrugated acoustic wave front. 


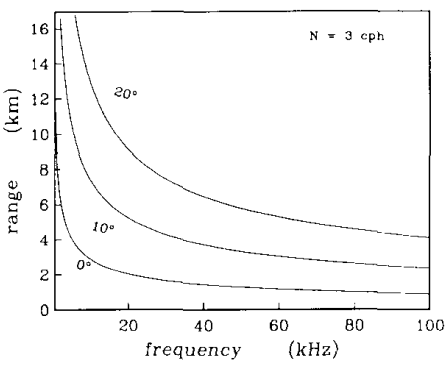

Fig. 1. These are the approximate limits of validity of the Rytov theory within the main thermocline. The calculations utilize the Garrett-Munk mid-latitude internal-wave refractive index model $F_{g}$ and the idealized situation of no focusing from a sound channel. The curves are loci of $\sigma_{I}^{2}=0.3$, the approximate limit of validity. Below the curves (at short ranges and/or lower frequency) the theory is valid. Three cases are shown in this figure: Propagation at $0^{\circ}$ (horizontal), $10^{\circ}$, and $20^{\circ}$, through typical stratification of the upper main thermocline ( 100 to $500 \mathrm{~m}$ depth), $N(z)=3$ cycles $/ \mathrm{h}$. Even at the most sensitive angle of $0^{\circ}$, the theory should work at 2 $\mathrm{km}$ for $20 \mathrm{kHz}$.

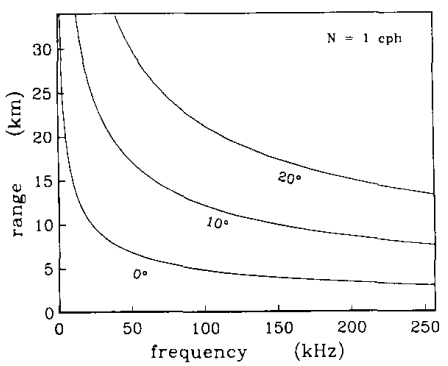

Fig. 2. Similar to Fig. 1, except for the deeper main thermocline, 500 to $1500 \mathrm{~m}$. The change in stratification to $N=1 \mathrm{cycle} / \mathrm{h}$, results in an increase of local vertical wavelengths of internal waves in the vertical modal model [20], [21], reducing vertical strain. The scintillations are reduced orders of magnitude relative to the $N=3 \mathrm{cph}$ case. The region of validity is quite large, extending to $10 \mathrm{~km}$ for $20 \mathrm{kHz}$ idealized horizontal transmission.

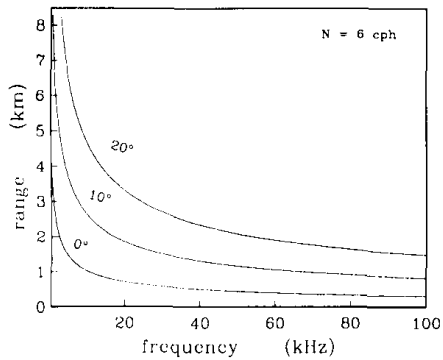

Fig. 3. Similar to Fig. 1, except for the near-surface region above a few hundred meters depth, $N=6 \mathrm{cph}$. Our perturbation theory calculations are not useful for horizontal transmission over $1 \mathrm{~km}$, but can be used up to a few kilometers for steeper transmission angles, exceeding $10^{\circ}$ from the horizontal.

At $\xi=0$ and $N(z)=N_{0}$, the intensity variance form internal waves follows the formula $\sigma_{I}^{2}=3.6 \times 10^{-9} \sigma R^{2}$, where $\sigma$ is the acoustic frequency in hertz and $R$ is the range in meters. Table I shows the relative scintillation indices for the situations of Figs. 1-3. An example of a situation beyond the strict usefulness of the Rytov theory is the MATE experiment [15], with $18 \mathrm{~km}$ transmission at $2 \mathrm{kHz}, N=1.8 \mathrm{cph}$, for which we estimate $\sigma_{I}^{2}=0.5$ (an invalid calculation), with measured $\sigma_{I}^{2}=$ 0.7 .

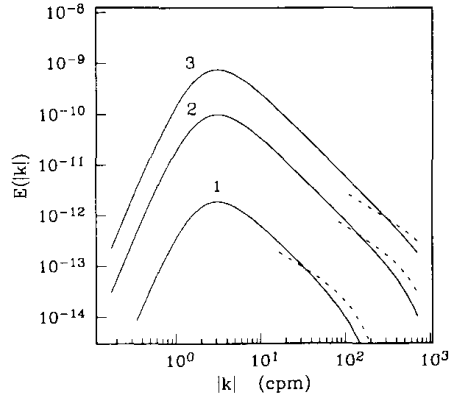

Fig. 4. Wavenumber magnitude spectra of main thermocline temperature microstructure models $E_{k}(k)$, converted to refractive index units, are shown. Three models with different $\chi_{T}$ and $\epsilon$ levels are shown. The spectral levels of the $E_{k}$ are chosen by matching $E_{k}$ at about the Kolmogorov wavenumber, $k_{k}=2 \pi / l_{c}$, with the appropriate Batchelor spectrum $E_{b}\left(k, \chi_{T}\right)$, which is valid for wavenumbers greater than $k_{k}$. The Batchelor spectra are shown dotted, to the right. The ratio $\chi_{T} / \epsilon=6$ for these models, and $k_{l}=2.0 \mathrm{rad} \mathrm{m}{ }^{-1}$, so that eddies are small. The three models have: (1) $\epsilon=5 \epsilon_{0}$, taken to represent the mean turbulence level (actually weak, nonturbulent microstructure); (2) $\epsilon=2000 \epsilon_{0}$; and (3) $\epsilon=40000 \epsilon_{0}$.

TABLE I

Relative Scintillation Indices for Propagation Through the GM InTERnal. WAVE Medium $F_{g}$ For Various Stratification Values $N$ AND ANGLES $\xi$

\begin{tabular}{llll}
\hline \hline$N(z)$ & $\xi=0$ & $\xi=10^{\circ}$ & $\xi=20^{\circ}$ \\
\hline $6 \mathrm{cph}$ & 7.9 & 1.2 & 0.38 \\
$3 \mathrm{cph}$ & 1.0 & 6.6 & 0.05 \\
$1 \mathrm{cph}$ & 0.0036 & 0.0056 & 0.0018 \\
\hline
\end{tabular}

Units are linear relative to $N(z)=N_{0}=3 \mathrm{cph}, \xi=0$.

To the first order throughout the mid-latitude ocean, the scaling of scintillation index with internal wave energy is direct, since $\sigma_{I}^{2}$ scales with $\left\langle\mu_{0}^{2}\right\rangle$, which in turn scales with rms vertical displacement and internal wave kinetic energy in the GM model [1]. Since internal wave kinetic energy varies by about a factor of 10 in the mid-latitude ocean [31], so should $\sigma_{I}^{2}$. However, the refractive index variance $\left\langle\mu_{0}^{2}\right\rangle$ may in general vary with respect to internal-wave energy, since relative contributions of salinity and temperature to stratification may vary. This thermohaline effect is discussed in [1] and [17]. It can be neglected when working within mid-latitudes, and simply accounted for at high latitudes.

There are three reasons why microstructure contributes weakly to deep-sea long-range scattering relative to finestructure. Ocean turbulence and/or microstructure is intermittent and usually absent, microstructure contributes weak sound-speed gradients, and the scale $R_{f}$ is usually larger than the largest microstructure wavelengths. As $R_{f}$ decreases with decreasing range or acoustic wavelength, microscale effects increase. In practice, the acoustic frequency effect dominates because transmission at reduced ranges gives weak cumulative scattering. By modeling unrealistically strong microstructure, one can identify the situations where microscale effects would contribute.

Fig. 4 shows three microstructure models with small outer scales of the order of a meter, representative of turbulence with the largest eddies limited in size by the stratification [25]. Using these models, one can compare relative scintillation indices for transmission through thermocline turbulence and thermocline internal waves. Model 1 has weak microstructure, consistent with mean levels predicted and observed. An estimate of energy dissipation of internal waves with the GM79 spectrum is $\epsilon_{0}=$ 
$3.2 \times 10^{-11} \mathrm{~W} \mathrm{~kg}^{-1}[32]$, but model 1 has $\epsilon$ five times higher, closer to measured values [33]. Models 2 and 3 have $\epsilon=2000 \epsilon_{0}$ and $\epsilon=40000 \epsilon_{0}$, which is quite strong turbulence, usually limited to regions of the ocean less than $10 \mathrm{~m}$ in vertical extent and a few hundred meters horizontally, not continuous as we model here. The ratio $\chi_{T} / \epsilon=6$ for these models, where $\chi_{T}$ is the dissipation rate of temperature, in $\mathrm{K}^{2} \mathrm{~s}^{-1}$. This ratio is related to the vertical mixing efficiency (fractional conversion of kinetic energy to potential energy) times the vertical temperature gradient [34] and is of interest. The relationships between internal wave decay, tubulence generation, vertical homogenization, and vertical heat flux are central concerns of small-scale ocean physics research.

Model 1 yields $\sigma_{I}^{2}<0.01$ for all ranges less than $20 \mathrm{~km}$ and frequencies less than $40 \mathrm{kHz}$. Model 2 yields $\sigma_{I}^{2} \approx 0.3$ for 10 $\mathrm{km}$ at $40 \mathrm{kHz}$, and for $15 \mathrm{~km}$ at $30 \mathrm{kHz}$, weaker scintillation than virtually all the fine structure cases shown in Figs. 1-3. Model 3 yields $\sigma_{I}^{2} \approx 0.3$ for $1 \mathrm{~km}$ at $40 \mathrm{kHz}, 5 \mathrm{~km}$ at $20 \mathrm{kHz}$, and $20 \mathrm{~km}$ at $10 \mathrm{kHz}$. The requirement of unrealistically high turbulence to achieve significant scintillation shows the lack of importance of microstructure for deep ocean transmission.

\section{Coastal and Mixing-Layer Transmission}

The two primary reasons that microstructure effects are minimal in the deep ocean are the gap between $R_{f}$ and the turbulence outer scale, and the weakness and intermittency of the turbulence. Attenuation at high frequency makes transmission over significant range (enough to integrate sufficient microstructure) at small $R_{f}$ impractical.

In coastal channels subject to strong tidal flow and in weakly stratified wind-driven surface mixing layers, however, the turbulence can be stronger and can extend to larger scale, overcoming both effects. In fact, scattering by surface-layer turbulence [2] and coastal-channel turbulence [19] has been found capable of exceeding perturbation theory limits at short range. We can simulate these situations with our homogeneous microstructure model $F_{k}$ if we reduce $k_{t}$, extending the model spectrum to larger length scales. We must assume here that a single strong path exists between the source and receiver (i.e., no boundary reflection or boundary reflections removed) or that multipath variations are coherent.

Two values of outer scale are used, $k_{t}=0.6$ and $k_{t}=2 \mathrm{rad}$ $\mathrm{m}^{-1}$. The smaller value allows very large eddies, $10 \mathrm{~m}$ or more, while the larger value limits them below $10 \mathrm{~m}$. From ocean mixing-layer measurements, we use spectral level $\phi_{t}$ from $8 \times$ $10^{-12}$ to $5 \times 10^{-9}$, corresponding to energy dissipation values $3 \times 10^{-9}<\chi_{T}<6 \times 10^{-6} \mathrm{~K}^{2} \mathrm{~s}^{-1}$ [34], [25], and we use the small eddy case, $k_{t}=2$. For a shallow turbulent coastal region the same dissipation levels will be used, but the larger outer scale will be used, $k_{t}=0.6$, allowing eddies which fill a shallow water column.

Vertical wavenumber spectra of intensity are always similar in shape for these models, with a flat region changing to a red region as $m$ increases. Representative spectra are shown in Fig. 5.

A parameter which is very influential and which was left constant for deep ocean simulation is the ratio $\chi_{T} / \varepsilon$. This ratio, associated with the efficiency of mixing for turbulence in a stratified fluid, varies widely in mixed-layer measurements [34]. In addition, if the wind-driven mixing layer is approximated as turbulent and is essentially unstratified, this ratio may reflect the entrainment of dense fluid at the base of the layer. The associa-
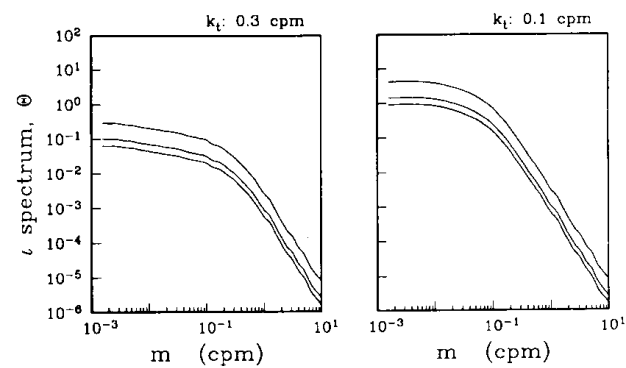

Fig. 5. One-dimensional transverse wavenumber spectra of intensity perturbations after propagation through homogeneous, isotropic, band-limited turbulence model $F_{k}$. Two outer scale cutoffs $k$, are used for the two frames, with smaller large-eddy size on the left $\left(k_{c}=2.0 \mathrm{rad} \mathrm{m}^{-1}\right)$ and larger large-eddy size on the right $\left(k_{t}=0.6 \mathrm{rad} \mathrm{m}^{-1}\right)$. For all spectra, the range is $4 \mathrm{~km}$, frequency $\sigma$ is $20 \mathrm{kHz}$, and $\chi_{T} / \epsilon=5$. Three thermal variance dissipation values are shown, from the top: $\chi_{T}=5 \times 10^{-7}$ $\chi_{T}=1 \times 10^{-7}$, and $\chi_{T}=5 \times 10^{-8} \mathrm{~K}^{2} / \mathrm{s}$.

tions of small-scale kinetic energy dissipation, mixing efficiency, and vertical heat flux are fundamental.

Scintillation indices for the schematic mixing-layer simulation, $k_{t}=2$, are shown in Fig. 6. Range, frequency, $\chi_{T}$, and $\chi_{T} / \epsilon$ are all allowed to vary. Fig. 7 shows the scintillation indices for the larger eddy case, $k_{t}=0.6$. Fig. 8 shows the standard deviation of phase, in radians, for the same set of ocean models but for only the $20 \mathrm{kHz}$ cases. The rms phase fluctuations are a few tenths of radians, or $20^{\circ}$ to $30^{\circ}$, for the largest scintillation situations that the theory can handle.

Fig. 9 shows transverse wave-number phase-fluctuation spectra for some of the situations summarized in Fig. 8. At a $10 \mathrm{~m}$ wavelength, the spectrum begins to descend, indicating relative stability of phase at a few meters separation with respect to separations greater than $10 \mathrm{~m}$.

Temperature (or sound speed) dissipation rate $\chi_{T}$, outer scale $k_{t}$, and $\chi / \epsilon$ all have strong influence on acoustic fluctuation statistics. The wide range of expected variability in these quantities makes calculations from even the simple perturbation model difficult to interpret, showing the prediction of scattering to be an essentially geophysical problem. The sensitivity to realistic variability within the family of microstructure models illustrates that one must prepare for a wide range of signal characteristics and quality in this regime.

\section{Conclusions}

Numerical calculation of acoustic field perturbation expressions can be used to predict fluctuations after propagation through ocean sound-speed structures, but before the onset of multipath. The general form of the expressions for signal spectra or correlation functions allow numerical evaluation for an unlimited quantity of vector wave-number spectral models of refractive index. In order to help define the bounds of applicability of the theory, log-intensity fluctuation variances have been calculated for three major situations: Ocean internal waves, ocean turbulence, and continuous strong large-scale turbulence.

Propagation through ocean thermocline internal waves, realistically weak thermocline turbulence, and unrealistically strong turbulence show that scintillations of intensity (and also of phase, although not shown) can be predicted and understood to first order up to ranges of tens of kilometers, given the proper transmission geometry. Internal wave effects dominate over any effects from expected microstructure. Nonhorizontal transmission yields small fluctuations, but eventually refractive effects of 

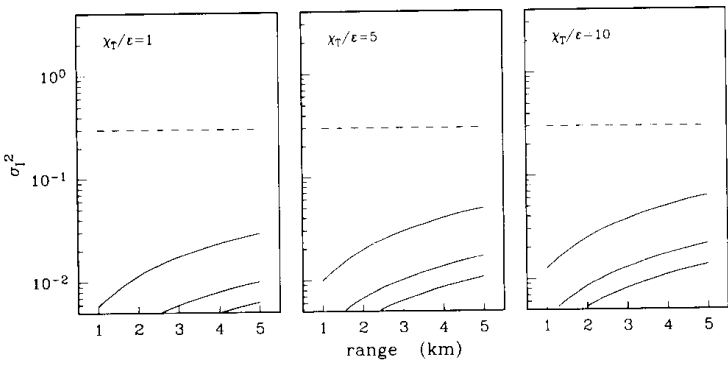

(a)
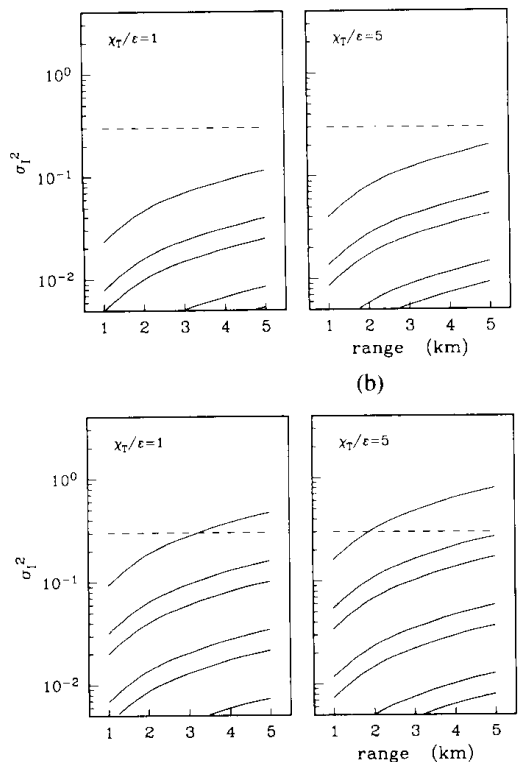

(c)

Fig. 6. Scintillation indices, as a function of range, for simulations through a mixing layer of limited activity or limited depth, with $k_{t}=2.0 \mathrm{rad} \mathrm{m}^{-1}$. Three frequencies are shown: (a) $10 \mathrm{kHz}$, (b) $20 \mathrm{kHz}$, and (c) $40 \mathrm{kHz}$. From left to right, three families of the thermal-to-kinetic energy dissipation ratio are shown. Simulations for various $\chi_{T}$ are shown within each frame, with $\chi_{T}=5 \times 10^{-6} \mathrm{~K}^{2} / \mathrm{s}$ for the uppermost curve, and with $\chi_{T}$ reduced by a factor of two for each descending curve. The dashed lines show the upper limit of strict applicability of the calculations, $\sigma_{I}^{2}=0.3$.

the sound channel will contribute some additional spatial variability and multipath, complicating the use of the theory.

Multipath due to the sound channel can exist at ranges where the random small-scale structures would contribute only small perturbations (no multipath from small structures). Interference between those signals is not described by this theory, but directional transmitters and receivers could attenuate the multipath effects. The multiple signals may also have passed through a limited-enough region of the ocean so that random finestructure effects are coherent between the distinct ensonified ducts or ray tubes. This coherent multipath behavior (the partial saturation regime) may serve to explain the success of Rytov phase modeling over Rytov amplitude modeling [35].

The relative consistency of deep ocean internal waves contrasts with the coastal situation measured by Farmer et al. [19] and modeled here with a turbulent ocean having a limited eddy size, where the limit may mimic the depth of the water. The many possibilities of outer scale, dissipation (mean-squared
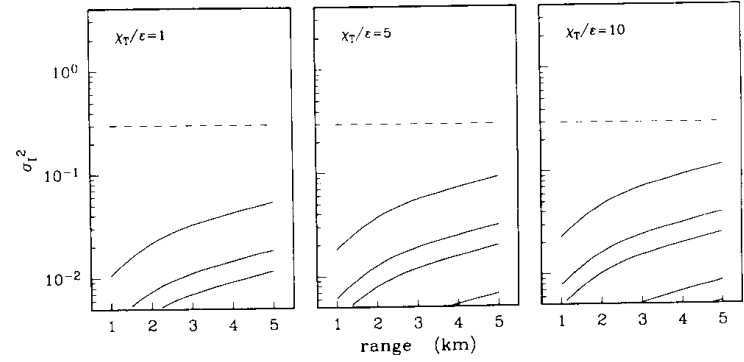

(a)
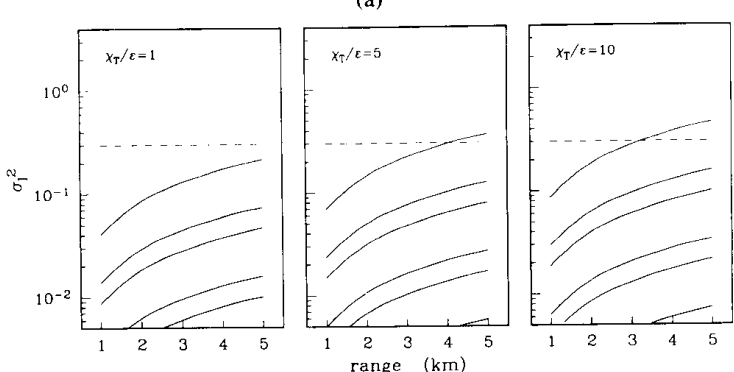

(b)
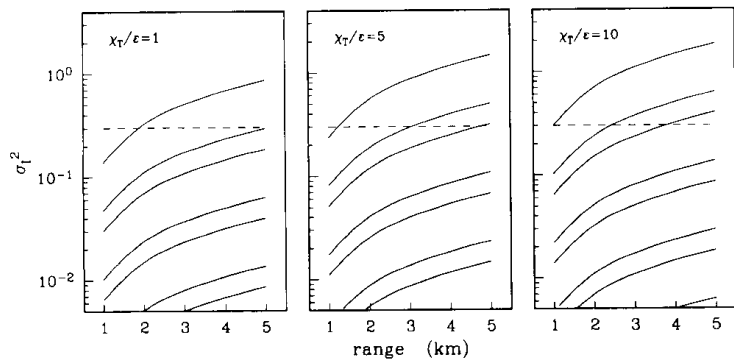

(c)
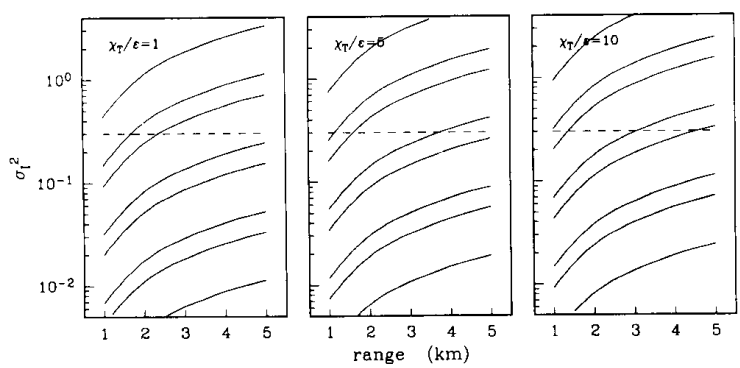

(d)

Fig. 7. Scintillation indices for simulations through a mixing layer of larger large eddies than that of Fig. $6, k_{t}=0.6 \mathrm{rad} \mathrm{m}^{-1}$. The larger eddies give stronger scintillations because the thermal fluctuations are not limited to sizes much smaller than $R_{f}$. Four frequencies are shown: (a) $5 \mathrm{kHz}$, (b) 10 $\mathrm{kHz}$, (c) $20 \mathrm{kHz}$, and (d) $40 \mathrm{kHz}$. The ratio $\chi_{T} / \epsilon$ and $\chi_{T}$ vary as in Fig. 6 . If a constant outer scale $k_{t}$ is considered, the scintillation index appears to be a simple function of both the propagation parameters (range and frequency) and the turbulence parameters. As in Fig. 6, values above the dashed line are uncertain.

small-scale shear), and ratio of thermal-gradient-variance-to-dissipation $\left(\chi_{T} / \epsilon\right)$ leave an inconclusive result for the phase and log-amplitude variance predictions and also for the estimates of the useful ranges for the theory. It is probable that intensity fluctuations may be order-unity relative to the mean (and thus out of bounds for this theory) for a modest $1 \mathrm{~km}$ at $20 \mathrm{kHz}$ (Fig. 

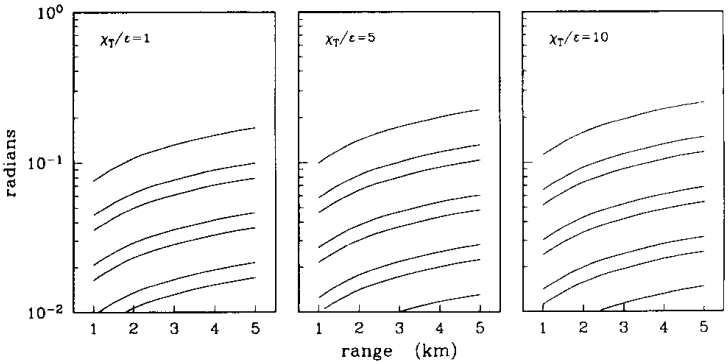

(a)
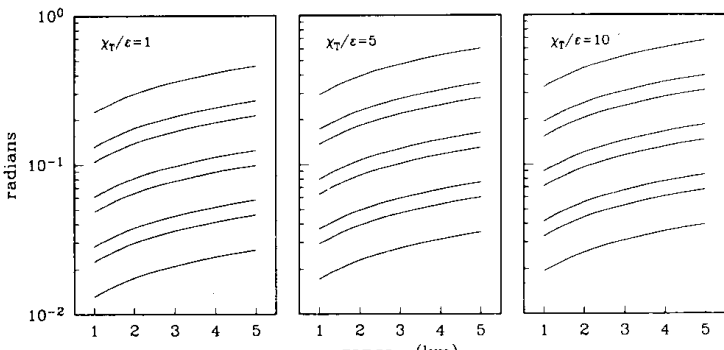

(b)

Fig. 8. Standard deviation of phase $\phi$ for simulations with some of the same $F_{k}$ models utilized for Figs. 6 and 7 . Results for both small eddies (a) and large eddies (b) are shown. The ratio $\chi_{T} / \epsilon$ and $\chi_{T}$ vary as in Fig. 6. Only the $20 \mathrm{kHz}$ case is shown. Simulations for various $\chi_{T}$ are shown within each frame, with $\chi_{T}=5 \times 10^{-6} \mathrm{~K}^{2} / \mathrm{s}$ for the uppermost curve, and with $\chi_{T}$ reduced by a factor of two for each descending curve. The highest curves in Fig. 8(a) are subject to errors, in the manner of those in Fig. 7(c).
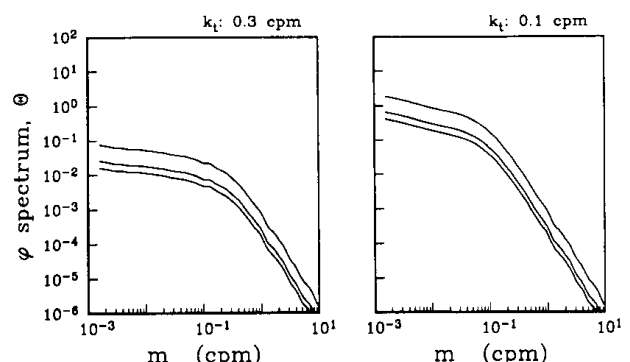

Fig. 9. One-dimensional transverse wavenumber spectra of phase perturbations. These are the same situations as in Fig. 5. Two outer scale cutoffs $k$, are used, with the smaller eddy size on the left $\left(k_{t}=2.0 \mathrm{rad} \mathrm{m}^{-1}\right)$, and larger eddy size on the right $\left(k_{t}=0.6 \mathrm{rad} \mathrm{m}^{-1}\right)$. For all spectra, the range is $4 \mathrm{~km}, \sigma=20 \mathrm{kHz}$, and $\chi_{T} / \epsilon=5$. Three thermal variance dissipation values are shown, from the top: $\chi_{T}=5 \times 10^{-7}, 10^{-7}$, and $5 \times 10^{-8}$ $\mathrm{K}^{2} / \mathrm{s}$.

7(c)). However, it is also probable for areas to exist with weak and predictable fluctuations at $40 \mathrm{kHz}$ and $3 \mathrm{~km}$, with phase and amplitude fluctuations falling within the Rytov regime for any expected variations of the medium spectrum (Fig. 6(b)). Three parameters of the medium are poorly defined for the strong microstructure situation: The outer scale of energetic turbulence, or the largest eddy size; the mean-squared gradient of temperature; and the mean-squared gradient of velocity, $\epsilon$.

\section{REFERENCES}

[1] T, F. Duda, S. M. Flatté, and D. B. Creamer, "Modeling meter-scale acoustic intensity fluctuations from oceanic fine structure and microstructure," J. Geophys. Res., vol. 93, pp. 5130-5142, 1988.

[2] T. F. Duda and S. M. Flatté, "Remote sensing of ocean turbulence using unsaturated acoustic transmission," in Proc. 8th
Symp. Turbulence and Diffusion, Amer. Meteor. Soc., 1988, pp. $168-171$

[3] S. M. Flatté, R. Leung, and S. Y. Lee, "Frequency spectra of acoustic fluctuations caused by oceanic internal waves and other finestructure," J. Acoust. Soc. Amer., vol. 68, pp. $1773-1779,1980$

[4] M. Schulkin, "Intensity variance of spherical waves in anisotropic media," J. Acoust. Soc. Amer., vol. 73, pp. 1187-1191, 1983.

[5] H. Medwin, "Sound phase and amplitude fluctuations due to temperature microstructure in the upper ocean," $J$. Acoust. Soc. Amer., vol. 56, pp. 1105-1110, 1974.

[6] V. I. Tatarskii, The Effects of the Turbulent Atmosphere on Wave Propagation. Springfield, VA: Nat. Tech. Info. Ser., 1971.

[7] S. F. Clifford, "The classical theory of wave propagation in a turbulent medium," in Laser Beam Propagation in the Atmosphere, J. W. Strohbehn, Ed. Berlin: Springer-Verlag, 1978.

[8] J. W. Strohbehn, "Modern theories in the propagation of optical waves in a turbulent medium," in Laser Beam Propagation in the Atmosphere, J. W. Strohbehn, Ed. Berlin: Springer-Verlag, 1978.

[9] A. Ishimaru, Wave Propagation and Scattering in Random Media, vol. 2. Orlando, FL: Academic, 1978.

[10] B. J. Uscinski, "Analytic solution of the fourth-moment equation and interpretation as a set of phase screens," J. Opt. Soc. Amer. A, vol. 2, pp. 2011-2091, 1985.

[11] R. G. Frehlich, "Intensity covariance of a point source in a random medium with a Kolmogorov spectrum and an inner scale of turbulence,"' J. Opt. Soc. Amer. A, vol. 4, pp. 360-366, 1987.

[12] R. L. Fante, "Electromagnetic beam propagation in turbulent media,"' Proc. IEEE, vol. 63, pp. 1669-1692, 1975

[13] J. M. Martin and S. M. Flatté, "Simulation of point-source scintillation through three-dimensional random media," $J$. Opt. Soc. Amer. $A$, vol. 7, pp. 838-847, 1990.

[14] J. W. Goodman, Statistical Optics. New York: Wiley, 1985.

[15] T. E. Ewart and S. A. Reynolds, "The Mid-ocean Acoustic Transmission Experiment: MATE," J. Acoust. Soc. Amer., vol. 75 , pp. $785-802,1984$.

[16] W. H. Munk and F. Zachariasen, "Sound propagation through a fluctuating stratified ocean-theory and observation," $J$. Acoust. Soc. Amer., vol. 59, pp. 818-838, 1976.

[17] S. M. Flatté, R. Dashen, W. H. Munk, K. M. Watson, and F. Zachariasen, Sound Transmission Through a Fluctuating Ocean. New York: Cambridge Univ. Press, 1979.

[18] H. Tennekes and J. L. Lumley, A First Course in Turbulence. Cambridge, MA: MIT Press, 1972

[19] D. M. Farmer, S. F. Clifford, and J. A. Verrall, "Scintillation structure of a turbulent tidal flow," J. Geophys. Res., vol. 92, pp. 5369-5382, 1987.

[20] W. H. Munk, "Internal waves and small-scale processes," in Evolution of Physical Oceanography, B. A. Warren and C. Wunsch, Eds. Cambridge, MA: MIT Press, 1981, pp. 264-291.

[21] C. J. R. Garrett and W. H. Munk, "Space-time scales of internal waves," Geophys. Fluid Dyn., vol. 3, pp. 225-264, 1972.

[22] G. K. Batchelor, The Theory of Homogeneous Turbulence. New York: Cambridge Univ. Press, 1953.

[23] A. E. Gargett, P. J. Hendricks, T. B. Sanford, T. R. Osborn, and A. J. Williams III, "A composite spectrum of vertical shear in the upper ocean," $J$. Phys. Oceanogr., vol. 11, pp. $1258-1271,1981$.

[24] T. M. Dillon, "Vertical overturns: A comparison of Thorpe and Ozmidov length scales." $J$, Geophys. Res., vol. 87, pp. 9601-9613, 1982.

[25] M. C. Gregg, "Diapycnal mixing in the thermocline: A review," J. Geophys. Res., vol. 92, pp. 5249-5286, 1987.

[26] G. K. Batchelor, "Small-scale variation of convected quantities like temperature in a turbulent fluid," $J$. Fluid. Mech., vol. 5 , pp. $113-133,1959$.

[27] N. S. Oakey, "Determination of the rate of dissipation of turbulent energy from simultaneous temperature and velocity shear microstructure measurements," J. Phys. Oceanogr., vol. 12, pp. 256-271, 1982

[28] S. Frankenthal, "Close range scintillations in anisotropic scattering media," J. Acoust. Soc. Amer., vol. 77, pp. 1395-1402, 1985 . 
[29] R. B. Stoughton, S. M. Flatté, and B. M. Howe, "Acoustic measurements of internal wave rms displacement and rms horizontal current off Bermuda in late 1983," J. Geophys. Res. vol. 91 , pp. 7721-7732, 1986.

[30] S. M. Flatté and R. B. Stoughton, "Theory of acoustic measurement of internal wave strength as a function of depth, horizontal position, and time," J. Geophys. Res., vol. 91, pp. 7709-7720, 1986.

[31] M. R. Levine, C. Paulson, and J. Morison, "Internal waves in the Arctic Ocean: Comparison with lower-latitude observations," J. Phys. Oceanogr., vol. 15, pp. 800-809, 1985.

[32] F. S. Henyey, J. A. Wright, and S. M. Flatté, "Energy and action flow through the internal wave field: An eikonal approach," J. Geophys. Res., vol. 91, pp. 8487-8496, 1986.

[33] M. C. Gregg, E. A. D'Asaro, T. J. Shay, and N. Larson, "Observations of persistent mixing and near-inertial internal waves," J. Phys. Oceanogr., vol. 16, pp. 856-885, 1986.

[34] N. S. Oakey, "Statistics of mixing parameters in the upper ocean during JASIN phase 2," J. Phys. Oceanogr., vol. 15, pp. $1662-1675,1985$
[35] S. M. Flatté, "Wave propagation through random media: Contributions from ocean acoustics," Proc. IEEE, vol. 71, pp. 1267-1294, 1983.

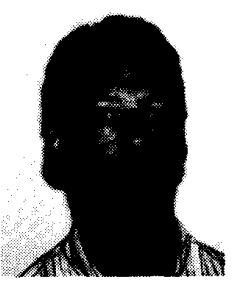

Timothy F. Duda received the B.A. degree in physics from Pomona College in 1979, and the Ph.D. degree in oceanography from Scripps Institution of Oceanography, University of California, San Diego, in 1986.

He has worked as a Geophysicist at the University of California, Santa Cruz since 1986.

His primary field of study is the measurement and characterization of ocean internal waves and microstructure. In addition to analyzing fluctuations of ocean acoustic transmissions, he has made electromagnetic-type internal-wave measurements, and in situ measurements of ocean microstructure.

Dr. Duda is a member of the American Meteorological Society, the American Geophysical Union, and the Acoustical Society of America. 